\title{
EXCESSO DE PESO INFLUENCIA NEGATIVAMENTE A CAPACIDADE FUNCIONAL DE IDOSOS EM ATIVIDADES COTIDIANAS
}

\author{
Overweight negatively influences functional \\ capacity of older people in daily activities
}

\author{
Luciana Bronzi de Souza ${ }^{\mathrm{a}, *}$ (C), Rafael Aiello Bomfimb ${ }^{\circ}$, \\ Amanda Gomes Macedo ${ }^{\complement} \odot$, Marcella Leite Mestrec $\left.{ }^{(}\right)$
}

OBJETIVO: Avaliar o estado nutricional, as condições de saúde e suas influências na capacidade funcional de idosos de um centro de convivência em Campo Grande, Mato Grosso do Sul. MÉTODOS: Trata-se de um estudo transversal, de base populacional, com 47 idosos de ambos os sexos, com idade entre 60 e 96 anos. A capacidade funcional foi definida por meio das atividades básicas e instrumentais da vida diária, operacionalizadas pelo índice de Katz e pela escala de Lawton e Brody. Foram aplicados um questionário sociodemográfico e dois recordatórios de 24 horas para avaliação do consumo alimentar; e foram avaliados a força de preensão manual e o estado nutricional. Um modelo de equação estrutural foi empregado para analisar a relação e as associações entre as variáveis. RESULTADOS: A amostra mostrou boa funcionalidade para as atividades básicas da vida diária, mas uma frequência alta de prejuízos nas atividades instrumentais, principalmente para tarefas como cuidar das finanças, preparar a própria refeição, limpar e realizar pequenos reparos domésticos. A baixa funcionalidade vai ao encontro da baixa força de preensão palmar encontrada. A maior parte dos idosos foi classificada como eutrófica (51,06\%). Ao aplicar o modelo de equação estrutural, obteve-se um coeficiente padronizado de 0,45; indicando efeito médio e significativo $(p=0,02)$ do índice de massa corporal sobre a dependência para as atividades básicas, com efeito direto. CONCLUSÃO: O excesso de peso influencia negativamente a capacidade funcional de idosos para desempenhar atividades básicas da vida diária.

PALAVRAS-CHAVE: envelhecimento; antropometria; força muscular; índice de massa corporal; força da mão; atividades cotidianas.

OBJECTIVE: To evaluate nutritional status, health conditions, and their impact on the functional capacity of aging adults in a community center in Campo Grande, state of Mato Grosso do Sul. METHODS: This is a cross-sectional, population-based study with 47 participants of both sexes, aged 60-96 years. Functional capacity was defined by the basic and instrumental activities of daily living, operationalized by the Katz index and the Lawton and Brody scale. A sociodemographic questionnaire and two 24-hour reminders were used to evaluate food consumption; hand grip strength and nutritional status were also evaluated. A structural equation model was used to analyze the relationship and associations between variables. RESULTS: The sample showed good functional capacity for the basic activities of daily living, but there was a high frequency of losses in instrumental activities, especially for tasks such as managing finances, cooking, cleaning and performing small domestic repairs. Low functional capacity is in agreement with the low hand grip strength found. Most participants were classified as eutrophic (51.06\%). When applying the structural equation model, a standardized coefficient of 0.45 was obtained; indicating a mean and significant effect ( $p=0.02$ ) of body mass index on dependence for basic activities, with direct effect. CONCLUSION: Overweight negatively influences the functional capacity of older adults to perform basic activities of daily living.

KEYWORDS: aging; anthropometry; muscle strength; body mass index; hand strength; activities of daily living.

aFaculdade de Nutrição, Universidade Federal de Goiás - Goiânia (GO), Brasil.

'Faculdade de Odontologia, Universidade Federal de Mato Grosso do Sul - Campo Grande (MS), Brasil.

'Faculdade de Ciências Farmacêuticas, Alimentos e Nutrição, Universidade Federal de Mato Grosso do Sul - Campo Grande (MS), Brasil.

Dados para correspondência

Luciana Bronzi de Souza - Faculdade de Nutrição, Universidade Federal de Goiás - Rua 227, Qd. 68, s/n. - Setor Leste Universitário - Campus I CEP: 74605-080 - Goiânia (GO), Brasil. E-mail: luciana.bronzi@gmail.com

Recebido em: 10/03/2019. Aceito em: 10/04/2019

DOI: $10.5327 / 22447-211520191900017$ 


\section{INTRODUÇÃO}

O envelhecimento é um fenômeno marcado por declínio funcional e perda de autonomia. As mudanças corporais decorrentes desse período, consideradas normais, como diminuição progressiva da massa muscular e aumento da quantidade de tecido adiposo levam ao decréscimo da capacidade funcional. ${ }^{1}$ Esse processo pode ser acelerado por diversos fatores, dentre eles, aspectos nutricionais, presença de múltiplas doenças crônicas e inatividade física, afetando de forma negativa o desempenho de atividades da vida diária. ${ }^{2}$

A capacidade funcional, avaliada por meio da realização das atividades da vida diária, é componente-chave para a avaliação da saúde dos idosos, uma vez que se relaciona à sua adaptação no ambiente social. Quando há comprometimento da capacidade funcional, há implicações para o idoso, a família e a comunidade, como maior probabilidade de quedas, fraturas, incapacidades, dependência, hospitalizações recorrentes e aumento da mortalidade. ${ }^{3}$

A dificuldade ou necessidade de ajuda para realizar atividades cotidianas em algum domínio da vida, ou incapacidade funcional, pode ser mensurada sob a ótica de dois domínios: atividades básicas da vida diária (ABVD), ${ }^{4}$ relação de seis atividades, hierarquicamente relacionadas, composta de atividades como vestir-se, banhar-se, alimentar-se; e atividades instrumentais da vida diária (AIVD), ${ }^{5}$ relacionadas à independência do indivíduo na sociedade, como, por exemplo, fazer compras, preparar refeições e utilizar meio de transporte.

Dificuldade em preparar suas próprias refeições e em se alimentar, situações comumente relacionadas à incapacidade funcional, podem levar à menor ingestão de alimentos e, consequentemente, comprometer o estado nutricional (EN) dos idosos. ${ }^{6}$

A alteração no EN é outro problema comum na terceira idade. A desnutrição, considerada um dos distúrbios mais comuns nessa fase da vida, pode trazer como consequência a diminuição da força muscular, contribuindo ainda mais para a incapacidade funcional. ${ }^{7}$ Já sobre o excesso de peso, há pouco na literatura que o relacione com o desempenho de atividades da vida diária.

Desta forma, o presente estudo teve como objetivo avaliar a influência do EN na capacidade funcional de idosos frequentadores de um centro de convivência de idosos de Campo Grande, Mato Grosso do Sul, além de conhecer o perfil nutricional, condições de saúde e de autonomia dessa população.

\section{MÉTODOS}

Trata-se de um estudo epidemiológico transversal, descritivo, de base populacional, realizado em um centro de convivência de idosos no município de Campo Grande, Mato Grosso do
Sul. São cadastrados na unidade 284 idosos. Foram excluídos indivíduos com idade inferior a 60 anos, os que não concluíram o protocolo do estudo na íntegra e aqueles com doenças incapacitantes (paralisia, acidente vascular cerebral, dificuldades de deambular ou também de responder ao questionário). A coleta ocorreu no período de junho a outubro de 2017.

Para avaliar a capacidade funcional, utilizou-se a escala de $\mathrm{Katz}^{4}$ para mensurar as ABVD e a escala de Lawton e Brody ${ }^{5}$ para mensurar as AIVD. Os idosos foram classificados como independentes caso não relatassem a necessidade de ajuda para realizar alguma ABVD e AIVD; e dependentes caso relatassem necessidade de ajuda em, pelo menos, uma das atividades de cada dimensão. Conforme proposto por Hoeymans et al., ${ }^{8}$ foi construída uma escala de incapacidade funcional hierárquica distinguindo três categorias:

- independentes;

- dependentes nas AIVD;

- dependentes nas ABVD e AIVD.

Os idosos que relataram dependência nas $\mathrm{ABVD}$, mas não nas AIVD, foram classificados na última categoria, referentes à dependência em ambas as dimensões.

A avaliação antropométrica consistiu na mensuração de peso, estatura, circunferência da cintura, braço e panturrilha. ${ }^{9,10}$ As medidas de circunferência foram realizadas com o auxílio de fita métrica inelástica, com precisão de $1 \mathrm{~mm}$. O índice de massa corporal (IMC) foi obtido pelo resultado da razão entre o peso corporal em quilogramas e a altura em metros elevada ao quadrado $\left(\mathrm{IMC}=\right.$ peso/altura $\left.{ }^{2}\right)$. Foi utilizada a classificação do estado nutricional de idosos proposta por Lipschitz, ${ }^{11}$ sendo baixo peso (menor ou igual a $22 \mathrm{~kg} / \mathrm{m}^{2}$ ), eutrofia (maior que $22 \mathrm{~kg} / \mathrm{m}^{2}$ e menor que $27 \mathrm{~kg} / \mathrm{m}^{2}$ ) e sobrepeso (maior ou igual a $27 \mathrm{~kg} / \mathrm{m}^{2}$ ). Para a avaliação da circunferência da cintura (CC), foram utilizados os pontos para classificação da obesidade abdominal, a saber: $>102 \mathrm{~cm}$ para homens e $>88 \mathrm{~cm}$ para mulheres. Para a classificação da circunferência da panturrilha $(\mathrm{CP})$, foram utilizados os valores que consideram adequada a circunferência igual ou superior a $31 \mathrm{~cm}$ para ambos os sexos. Valores inferiores são indicativos de risco de desnutrição em idosos.

Foi mensurada a força de preensão palmar por meio de dinamômetro manual hidráulico. Os dinamômetros foram calibrados antes do início da coleta dos dados. Os testes foram realizados na posição recomendada pela Sociedade Americana de Terapeutas de Mão (SATM).

Para a avaliação do consumo alimentar, foram aplicados dois recordatórios de 24 horas (R24h), em dias não consecutivos. Para melhor validade dos dados, o R24h foi empregado utilizando o método de passagem múltipla. Foram registrados: 
tipo de alimento, forma de preparo, local e horário de consumo e quantidade em medidas caseiras, com auxílio de utensílios e álbum fotográfico para garantir a padronização da quantidade consumida. ${ }^{12}$ Os dados de consumo foram obtidos com auxílio do software de análise de dietas Dietpro ${ }^{\circledR}$, versão 5.5i, Agromídia Ltda (Viçosa, Minas Gerais, Brasil). Os nutrientes considerados para a avaliação dos recordatórios foram: carboidratos, proteínas totais, lipídios totais, fibra alimentar total, cálcio e ferro, os quais tiveram seus valores comparados com as recomendações diárias de ingestão das Dietary Reference Intakes (DRI), para indivíduos de ambos os sexos, para a faixa etária correspondente.

Para conhecer o perfil social, econômico, demográfico e de saúde dos idosos, foi aplicado um questionário estruturado na forma de entrevista. $O$ protocolo incluiu os seguintes itens, que constituíram as variáveis independentes: sexo, idade (em anos completos), escolaridade (nível de instrução), profissão, estado civil (solteiro, casado, divorciado, viúvo), prática de atividade física, etnia autorreferida (caucasiano, afrodescendente, asiático) e condições de saúde (presença de patologias autorreferidas, hospitalização, queda e autoavaliação da saúde).

A equipe de entrevistadores foi submetida a treinamento prévio para aplicação do questionário, realização da entrevista e avaliação nutricional; e foi composta por nutricionistas e acadêmicos de nutrição.

Os resultados são apresentados por meio da estatística descritiva, como média aritmética, desvio padrão e porcentagem. Para as variáveis de consumo alimentar, como os dados apresentaram curva normal (Kolmogorov-Smirnov), foi utilizada estatística paramétrica, sendo utilizado o teste ANOVA, seguido do teste de Tukey. Um modelo de equação estrutural (SEM) foi empregado para analisar a relação estrutural e as associações entre as variáveis. Os coeficientes padronizados (SC) foram interpretados de acordo com Kline: ${ }^{13}$ um SC de 0,10 indica efeito pequeno; $\mathrm{SC}>0,10 \mathrm{e}$ $<0,30$ indica efeito médio; e $\mathrm{SC}>0,50$ indica efeito forte. Em todos os testes, foram utilizados nível de significância de $5 \%$ ou p valor correspondente e intervalo de confiança de 95\% (IC95\%). As análises foram realizadas no software Stata, versão 14 (StataCorp., CollegeStation, Estados Unidos).

O estudo foi aprovado pelo Comitê de Ética em Pesquisa da Universidade Federal de Mato Grosso do Sul, sob parecer $n^{\circ}$ 1.997.354. Os idosos iniciaram a participação somente após a assinatura do Termo de Consentimento Livre e Esclarecido, seguindo a explicação da elaboração da pesquisa, bem como da informação de desistência livre, se assim desejassem, e com a garantia de anonimato e sigilo das informações prestadas, de acordo com a Resolução no 466/2012 do Conselho Nacional de Saúde.

\section{RESULTADOS}

Dos 284 idosos cadastrados no centro de convivência, 47 deles, de ambos os sexos, concluíram o protocolo do estudo na íntegra, sendo a maioria do sexo feminino (87\%). A idade dos participantes variou entre 60 e 96 anos. A etnia predominante foi caucasiana $(80,2 \%)$, seguida por afrodescendentes $(15,2 \%)$ e asiáticos (4,6\%). Quanto à escolaridade, a maioria relatou ter cursado o ensino fundamental, como descrito na Tabela 1. Hipertensão arterial sistêmica $(39,1 \%)$ e diabetes mellitus $(26,1 \%)$ foram as patologias mais referidas. A prevalência de independência nas ABVD foi elevada $(63,8 \%)$, no entanto, nas AIVD foi bastante baixa (4,3\%).

As análises não foram separadas por sexo, pois o número de homens idosos participantes foi baixo $(n=6)$.

Os dados da Tabela 2 mostram a distribuição dos idosos segundo capacidade funcional para as ABVD e AIVD. A grande maioria dos idosos se mostrou independente para as ABVD, não sendo necessária nenhuma ajuda para a sua execução. Já as AIVD, que são atividades mais complexas, demandaram mais ajuda para a sua execução por parte da amostra avaliada.

O IMC médio da amostra foi $29,1 \mathrm{~kg} / \mathrm{m}^{2}$, variando de 14,1 a $48,1 \mathrm{~kg} / \mathrm{m}^{2}$; e sua classificação, bem como os demais dados antropométricos e de consumo alimentar, estão descritos na Tabela 3. A prevalência média de adequação do consumo de ferro foi $79,3 \%$; e de fibras, de $43,7 \%$.

Ao aplicar o SEM para analisar as relações das variáveis, obteve-se o coeficiente padronizado de 0,45 , indicando efeito médio e significativo $(\mathrm{p}=0,02)$ do IMC sobre a dependência para as $\mathrm{ABVD}$, com efeito direto. As demais variáveis analisadas, tais como circunferência da panturrilha $(p=0,582)$, do braço $(\mathrm{p}=0,923)$, força de preensão palmar direita e esquerda ( $p=0,169 ; p=0,220 ;$ respectivamente) e consumo alimentar (dados não apresentados) não exerceram efeito significativo na capacidade funcional dos idosos avaliados.

\section{DISCUSSÃO}

O presente trabalho objetivou avaliar a influência do estado nutricional na capacidade funcional de idosos frequentadores de um centro de convivência, além de conhecer seu perfil nutricional, condições de saúde e de autonomia. A maioria dos idosos foi considerada dependente, total ou parcialmente, principalmente no domínio instrumental.

Ao aplicar o SEM para análise do efeito que os aspectos nutricionais avaliados exerceram sobre a dependência de idosos, pôde-se notar a influência negativa do excesso de peso, avaliado pelo IMC (acima de $27 \mathrm{~kg} / \mathrm{m}^{2}$ ), na execução das $\mathrm{ABVD}$ dos idosos, com efeito médio e direto. Os demais 
Tabela 1 Características descritivas e de capacidade funcional de idosos de um centro de convivência. Campo Grande, Mato Grosso do Sul, 2017.

\begin{tabular}{|c|c|c|}
\hline Variáveis & n & $\%$ \\
\hline \multicolumn{3}{|l|}{ Número doenças crônicas } \\
\hline Nenhuma & 13 & 27,66 \\
\hline Uma & 13 & 27,66 \\
\hline Duas & 9 & 19,15 \\
\hline Três ou mais & 12 & 25,53 \\
\hline \multicolumn{3}{|l|}{ Atividade física } \\
\hline Sim & 43 & 91,49 \\
\hline Não & 4 & 8,51 \\
\hline \multicolumn{3}{|l|}{ Escolaridade } \\
\hline Analfabeto & 10 & 21,28 \\
\hline Ensino fundamental & 31 & 65,96 \\
\hline Ensino médio & 6 & 12,76 \\
\hline \multicolumn{3}{|l|}{ Estado civil } \\
\hline Solteiro(a) & 1 & 2,12 \\
\hline Casado(a) & 13 & 27,66 \\
\hline Separado(a) & 7 & 14,90 \\
\hline Viúvo(a) & 26 & 55,32 \\
\hline \multicolumn{3}{|l|}{ Perda de peso nos últimos 3 meses } \\
\hline Não sabe & 18 & 38,30 \\
\hline Entre 1 e 3 kg & 18 & 38,30 \\
\hline Superior a 3 kg & 11 & 23,40 \\
\hline \multicolumn{3}{|l|}{ Autoavaliação da saúde* } \\
\hline Pior & 4 & 8,51 \\
\hline Não sabe & 3 & 6,38 \\
\hline Igual & 14 & 29,79 \\
\hline Melhor & 26 & 55,32 \\
\hline \multicolumn{3}{|l|}{ Capacidade funcional } \\
\hline Independente & 2 & 4,25 \\
\hline Dependente em AIVD & 28 & 59,58 \\
\hline Dependentes em ABVD e AIVD & 17 & 36,17 \\
\hline
\end{tabular}

*Autoavaliação da saúde em comparação com outros idosos; AIVD: atividades instrumentais da vida diária; ABVD: atividades básicas da vida diária. aspectos nutricionais analisados não desempenharam efeito significativo na capacidade funcional dos participantes.

O envelhecimento populacional e o excesso de peso são problemas de saúde pública emergentes na atualidade. A transição demográfica, advinda do envelhecimento populacional, está diretamente relacionada à transição epidemiológica, em que há o aumento do número de doenças crônicas não transmissiveis (DCNT) e, dentre elas, destaca-se o aumento da prevalência de excesso de peso nessa população. ${ }^{14,15} \mathrm{O}$ excesso de peso entre idosos pode ser resultante de mudanças em sua dieta nas últimas décadas, com aumento do consumo de alimentos processados, ricos em açúcares e gorduras. ${ }^{16}$

Tabela 2 Independência de idosos de um centro de convivência, segundo atividades básicas da vida diária e atividades instrumentais da vida diária. Campo Grande, Mato Grosso do Sul, Brasil, 2017.

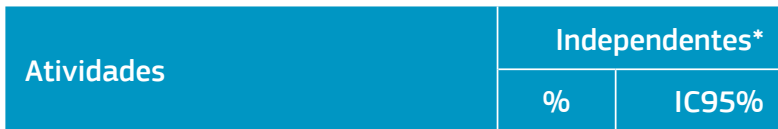

Atividades básicas da vida diária

\begin{tabular}{l|c|c}
\hline Banhar-se & 84,78 & $75,92-90,86$ \\
\hline Vestir-se & 84,78 & $75,92-90,86$ \\
\hline Usar o banheiro & 86,95 & $78,22-92,52$ \\
\hline Deitar-se/sentar-se & 89,13 & $80,77-94,12$ \\
\hline Controle de fezes e urina & 80,43 & $70,86-87,42$ \\
\hline Alimentar-se & 69,56 & $59,24-78,23$ \\
\hline Atividades instrumentais da vida diária & \\
\hline Usar o telefone & 76,08 & $66,13-83,82$ \\
\hline $\begin{array}{l}\text { Ir a lugares distantes, de } \\
\text { transporte, sem planejamento } \\
\text { especial }\end{array}$ & 76,08 & $66,13-83,82$ \\
\hline $\begin{array}{l}\text { Fazer compra } \\
\text { Preparar as próprias refeições }\end{array}$ & 4,34 & $1,61-11,20$ \\
\hline $\begin{array}{l}\text { Arrumar a casa } \\
\text { mazer pequenos reparos } \\
\text { domésticos }\end{array}$ & 2,17 & $0,05-8,48$ \\
\hline \begin{tabular}{l} 
Lavar e passar a própria roupa \\
\hline Cuidar das próprias finanças
\end{tabular} & 2,17 & $0,05-8,48$ \\
\hline
\end{tabular}

*Independência: capacidade de realizar atividades sozinho; IC95\%: intervalo de confiança de 95\%. 
A obesidade piora a função física de idosos, leva à fragilidade e dependência e compromete a qualidade de vida. Dada a crescente prevalência de obesidade, no futuro próximo, acredita-se que os indivíduos que apresentarão maior fragilidade serão os obesos, deficientes e adultos idosos. ${ }^{16}$ Segundo Santos et al., ${ }^{17}$ o sobrepeso e a obesidade associados ao processo de envelhecimento podem interferir na capacidade funcional nos idosos, causando diminuição de habilidades físicas, comprometimento do sistema motor e, portanto, na mobilidade para realização de algumas tarefas.

Nesse sentido, alguns trabalhos vêm apresentando diferentes resultados sobre a relação entre excesso de peso e diminuição da capacidade funcional em idosos. $\mathrm{O}$ excesso de peso e sua relação com a capacidade funcional foi avaliada por Sousa et al. ${ }^{7}$ em idosos institucionalizados. Nessa população, o excesso de peso foi pouco prevalente, diferentemente dos dados aqui descritos, e não interferiu na dependência dos idoso nas ABVD. ${ }^{4}$

Já em um estudo com idosas não institucionalizadas, que apresentavam sobrepeso, encontrou-se um índice de aptidão física abaixo do normal. No entanto, a metodologia para avaliar a capacidade funcional adotada por esses pesquisadores foi diferente da que utilizamos, sendo empregados instrumentos que avaliaram coordenação, resistência e agilidade física, variáveis que foram prejudicadas pelo excesso de peso. ${ }^{18}$

Oliveira et al., ${ }^{19} \mathrm{em}$ trabalho realizado com idosos de um grupo de convivência, avaliaram a correlação da massa corporal com o desempenho motor. Os autores encontraram diferença estatística significativa entre as variáveis IMC (acima de $\left.27 \mathrm{~kg} / \mathrm{m}^{2}\right)$ e limitação do equilíbrio $(p=0,008)$ e limitação da atividade de agachar e pegar um lápis no chão $(\mathrm{p}=0,004)$.

Tabela 3 Estado nutricional de idosos de um centro de convivência. Campo Grande, Mato Grosso do Sul, Brasil, 2017.

\begin{tabular}{|c|c|c|c|c|c|}
\hline Variáveis & Média & DP & Mín. & Máx. & IC95\% \\
\hline \multicolumn{6}{|l|}{ Medidas corporais } \\
\hline Peso (kg) & 69,96 & 15,61 & 31 & 114 & $66,76-73,17$ \\
\hline Estatura $(\mathrm{m})$ & 1,55 & 0,08 & 1,38 & 1,76 & $1,54-1,57$ \\
\hline FPPD (kg) & 16,72 & 0,60 & 7 & 30 & $15,51-17,92$ \\
\hline \multicolumn{6}{|l|}{ Dados dietéticos } \\
\hline Calorias totais (kcal) & $1.305,28$ & 495,66 & 408,72 & $2.840,72$ & $1.202,88-1.407,68$ \\
\hline Carboidratos (\%VET) & 52,22 & 15,09 & 12,51 & 85,02 & $49,10-55,34$ \\
\hline Proteínas (\%VET) & 23,65 & 11,13 & 5,21 & 90,67 & $21,23-25,95$ \\
\hline Proteína/kg/dia & 0,93 & 0,06 & 0,11 & 3,68 & 0,8137-1,0549 \\
\hline Lipídeos (\%VET) & 25,45 & 12,23 & 4,87 & 77,64 & $22,92-27,97$ \\
\hline Fibras (g) & 17,08 & 12,72 & 1,03 & 100,46 & $14,45-19,71$ \\
\hline \multicolumn{6}{|l|}{ IMC } \\
\hline Baixo peso (\%) & 4,25 & - & - & - & - \\
\hline Eutrofia (\%) & 51,06 & - & - & - & - \\
\hline Excesso de peso (\%) & 44,69 & - & - & - & - \\
\hline \multicolumn{6}{|l|}{$\mathrm{CP}$} \\
\hline Risco para desnutrição (\%) & 12,77 & - & - & - & - \\
\hline
\end{tabular}

\begin{tabular}{l|l|l|l|l|l}
\hline Baixo risco DCV (\%) & 19,15 & - & - & - & - \\
\hline Risco aumentado DCV (\%) & 17,02 & - & - & - & - \\
\hline Risco muito aumentado DCV (\%) & 63,83 & - & - & - & - \\
\hline
\end{tabular}

DP: desvio padrão; IC95\%: intervalo de confiança de 95\%; FPPD: força de preensão palmar direita; VET: valor energético total; IMC: índice de massa corporal; CP: circunferência de panturrilha; CC: circunferência da cintura; DCV: doenças cardiovasculares. 
$\mathrm{Na}$ avaliação da função de membros inferiores e superiores de idosos, medidos pela habilidade de tocar o pescoço, força da mão e levantar sozinho de uma cadeira, Tecchi e Gessinger ${ }^{14}$ não encontraram associação significativa entre o IMC e a funcionalidade de idosos. Segundo o autor, o excesso de peso e a desnutrição não interferiram na capacidade funcional de idosos, avaliada pela função dos membros inferiores e superiores.

$\mathrm{Na}$ literatura, é bastante discutida a relação da desnutrição com a perda da capacidade funcional. Idosos desnutridos apresentam maior dependência nas atividades de vida diária. ${ }^{20}$ A desnutrição, assim como o excesso de peso, é um problema comum na terceira idade e pode contribuir para o aumento da mortalidade, redução na qualidade de vida e tem como consequência a diminuição da força muscular e da capacidade de ação, contribuindo para a diminuição da capacidade funcional.

Entre as atividades de vida diária mais comprometidas em idosos desnutridos, ressalta-se o ato de se alimentar, ${ }^{21,22}$ assim como também encontrado no presente trabalho. Para os idosos dessa amostra, a atividade de se alimentar foi a que apresentou menor independência $(69,52 \%)$. Tal achado pode impactar sobremaneira o estado nutricional e funcional desses indivíduos, uma vez que, de acordo com os resultados aqui descritos e também os já descritos na literatura, a manutenção do peso normal parece ser o melhor diagnóstico nutricional para a preservação da capacidade funcional de idosos.

Apesar da dificuldade em alimentar-se, quando avaliado o consumo alimentar da amostra, a média de ingestão de proteínas por $\mathrm{kg}$ de peso foi de 0,93 gramas, valor superior à ingestão dietética recomendada (RDA) de $0,8 \mathrm{~g} / \mathrm{kg}$ de peso corporal por dia ${ }^{23}$ (IOM, 2006). No entanto, Valenzuela et al..$^{24}$ sugerem que a ingestão proteica seja de $1,2 \mathrm{~g} / \mathrm{kg} /$ dia para a redução de perda de massa muscular em idosos. Os dados de consumo proteico da amostra vão ao encontro do comprometimento da capacidade funcional. Uma oferta proteica satisfatória está associada à diminuição e prevenção da perda de massa muscular, reduzindo o fator de risco para fragilidade em idosos, além de auxiliar na melhora de funções físicas e mentais. ${ }^{25}$

Esses resultados devem ser considerados à luz de algumas limitações. Primeiramente podemos citar a baixa aderência dos idosos em participar da pesquisa, ocasionando assim uma amostra pequena. Ainda, por ter um desenho metodológico transversal, não coube a este estudo buscar explicações para a associação encontrada. É necessário que sejam realizados estudos longitudinais para se investigar mais detalhadamente as relações entre aspectos do estado nutricional de idosos e a capacidade funcional e, consequentemente, desenvolver estratégias de promoção da saúde e prevenção de doenças nessa população.

\section{CONCLUSÃO}

Os dados evidenciam que o IMC acima de $27 \mathrm{~kg} / \mathrm{m}^{2}$, classificado como excesso de peso, exerce efeito negativo sobre capacidade funcional de idosos em realizar tarefas cotidianas, podendo ocasionar problemas relacionados à diminuição da dependência, que estão associados à perda de autonomia e diminuição da qualidade de vida nessa faixa etária. Essa é uma importante condição que requer, por parte de profissionais de saúde da área, cuidado especializado, com ênfase na alimentação e nutrição.

\section{AGRADECIMENTOS}

Agradecemos à nutricionista Taiz Siqueira Pinto, o auxílio na coleta de dados; e à Universidade Federal de Mato Grosso do Sul, por permitir a condução do trabalho em seu Laboratório de Avaliação Nutricional.

\section{CONFLITO DE INTERESSES}

Os autores declaram não haver conflito de interesses.

\section{REFERÊNCIAS}

1. Giampaoli S, Ferrucci L, Cecchi F, Lo Noce C, Poce A, Dima F, et al. Hand-grip strength predicts incident disability in non-disabled older men. Age Ageing. 1999;28(3):283-8. https://doi.org/10.1093/ ageing/28.3.283

2. Alexandre TS, Duarte YAO, Santos JLF, Lebão ML. Relação entre força de preensão manual e dificuldade no desempenho de atividades básicas de vida diária em idosos do município de São Paulo. Saúde Coletiva. 2008;5(24):178-82.

3. Nunes AC, Oliveira LC, Wagner R. Identificação de anemia por carência de ferro em idosos residentes em instituições de amparo de Curitiba e região Metropolitana. Cad Esc Saúde [Internet]. 2011 [acessado em 22 out. 2018];5(1). Disponível em: http://portaldeperiodicos.unibrasil. com.br/index.php/cadernossaude/article/view/2319
4. Katz S, Ford AB, Moskowitz RW, Jackson BA, Jaffe MW. Studies of Illness in the Aged: The Index of ADL: A Standardized Measure of Biological and Psychosocial Function. JAMA [Internet]. 1963 [acessado em 26 out. 2017];185(12):914-9. Disponível em: https://doi.org/10.1001/ jama.1963.03060120024016

5. Lawton MP, Brody EM. Assessment of Older People: Self-Maintaining and Instrumental Activities of Daily Living. Gerontologist [Internet] 1969 [acessado em 26 out. 2017];9(3):179-86. Disponível em: https:// doi.org/10.1093/geront/9.3_Part_1.179

6. Santos KT, Santos Júnior JCC, Rocha SV, Reis LA, Coqueiro RS, Fernandes $\mathrm{MH}$. Indicadores antropométricos de estado nutricional como preditores de capacidade em idosos. Rev Bras Med Esporte [Internet]. 2014 [acessado em 13 fev. 2018];20(3):181-5. Disponível em: http://www. scielo.br/pdf/rbme/v20n3/1517-8692-rbme-20-03-00181.pdf 
7. Sousa KT, Mesquita LAS, Pereira LA, Azeredo CM. Baixo peso e dependência funcional em idosos institucionalizados de Uberlândia (MG), Brasil. Rev Ciênc Saúde Coletiva. 2014;19(8):3513-20. http:// dx.doi.org/10.1590/1413-81232014198.21472013

8. Hoeymans N, Feskens EJM, van den Bos GAM, Kromhout D. Measuring functional status: Cross-sectional and longitudinal associations between performance and self-report (Zutphen Elderly Study 1990-1993). J Clin Epidemiol [Internet]. 1996 [acessado em 28 out. 2017];49(10):110310. Disponível em: https://doi.org/10.1016/0895-4356(96)00210-7

9. Lohman TG, Roche AF, Martorell R. Anthropometric standardization reference manual. Champaign: Human Kinetics Books; 1988.

10. Silva DAS, Pelegrini A, Pires-Neto CS, Vieira MFS, Petroski EL. O antropometrista na busca de dados mais confiáveis. Rev Bras Cineantropometria e Desempenho Hum. 2011;13(1):82-5. http:// dx.doi.org/10.5007/1980-0037.2011v13n1p82

11. Lipschitz DA. Screening for nutritional status in the elderly. Prim Care. 1994;21(1):55-67.

12. Monteiro JP. Consumo alimentar : visualizando porções [Internet]. Rio de Janeiro: Grupo Gen - Guanabara Koogan; 2010 [acessado em 11 out. 2018]. Disponível em: http://www.editorametha.com.br/ livros-de-nutricao/nutricao-clinica/consumo-alimentar-visualizandoporcoes-serie-nutric-o-e-metabolismo.html

13. Kline P. An easy guide to factor analysis [Internet]. Routledge; 1994 [acessado em 28 out. 2017]. Disponível em: https://books.google.com.br/books/about/ An_Easy_Guide_to_Factor_Analysis.html?id=6PHzhLD-bSoC\&redir_esc $=y$

14. Tecchio JM, Gessinger C. Upper and lower limb functionality and body mass index in physically active older adults. Fisioter Mov. 2017;30(Supl. 1):45-54. http://dx.doi.org/10.1590/1980-5918.030.s01.ao04

15. Lima-Costa MF, Facchini LA, Matos DL, Macinko J. Mudanças em dez anos das desigualdades sociais em saúde dos idosos brasileiros (1998-2008) Rev Saúde Pública [Internet]. 2012 [acessado em 18 nov. 2017];46(Supl. 1). Disponível em: http://dx.doi.org/10.1590/50034-89102012005000059

16. Villareal DT, Chode S, Parimi N, Sinacore DR, Hilton T, ArmamentoVillareal R, et al. Weight loss, exercise, or both and physical function in obese older adults. N Engl J Med. 2011;364(13):1218-29. https:// doi.org/10.1056/NEJMoa1008234
17. Santos RR, Bicalho MAC, Mota P, Oliveira DR, Moraes EN. Obesity in the elderly. Rev Méd Minas Gerais. 2013;23(1):64-73. http://www. dx.doi.org/10.5935/2238-3182.20130011

18. Rech CR, Cruz JLS, Araújo EDS, Kalinowski FG, Dellagrana RA. Associação entre aptidão funcional e excesso de peso em mulheres idosas. Motri. 2010;6(2):47-53.

19. Oliveira TA, Duarte SFP, Reis LA. Relação entre índice de massa corporal e desempenho motor de idosos pertencentes a grupos de convivência. Texto Context Enferm. 2016;25(4). http://dx.doi.org/10.1590/010407072016003370014

20. Silva JL, Marques APO, Leal MCC, Alencar DL, Melo EMA. Fatores associados à desnutricão em idosos institucionalizados. Rev Bras Geriatr Gerontol. 2015;18(2):443-51. http://dx.doi.org/10.1590/18099823.2015.14026

21. Tannen A, Schütz T, Smoliner C, Dassen T, Lahmann N. Care problems and nursing interventions related to oral intake in German Nursing homes and hospitals: A descriptive mulitcentre study. Int J Nurs Stud. 2012:49(4):378-85. http://dx.doi.org/10.1016/j.jijnurstu.2011.09.018

22. Dion N, Cotart JL, Rabilloud M. Correction of nutrition test errors for more accurate quantification of the link between dental health and malnutrition. Nutrition. 2007;23(4):301-7. http://dx.doi.org/10.1016/j. nut.2007.01.009

23. Institute of Medicine. Dietary Reference Intakes: The Essential Guide to Nutrient Requirements Intakes DRI [Internet]. Washington, D.C. Institute of Medicine; 2006 [acessado em 14 fev. 2018]. Disponível em: http://www.nap.edu/catalog/11537.html

24. Valenzuela RER, Ponce JA, Morales-Figueroa GG, Muro KA, Carreón VR, Alemán-Mateo $\mathrm{H}$. Insufficient amounts and inadequate distribution of dietary protein intake in apparently healthy older adults in a developing country: implications for dietary strategies to prevent sarcopenia. Clin Interv Aging. 2013;8:1143-8. https://doi.org/10.2147/CIA.S49810

25. Beasley JM, Wertheim BC, LaCroix AZ, Prentice RL, Neuhouser ML, Tinker LF, et al. Biomarker-calibrated protein intake and physical function in the Women's Health Initiative. J Am Geriatr Soc [Internet]. 2013 [acessado em 14 fev. 2018];61(11):1863-71. Disponível em: https://doi.org/10.1111/jgs.12503 